\title{
Instrumental meat quality and fatty acid composition of lean muscle from beef steers offered grass silage alone or in combination with legume/cereal based wholecrop silage at two concentrate levels
}

P C Kennedy ${ }^{1,2}$, L E R Dawson ${ }^{2}$, B W Moss ${ }^{3}$, A Fearon ${ }^{3}$, D J Kilpatrick ${ }^{3}$

${ }^{1}$ Queens University, Belfast, United Kingdom, ${ }^{2}$ Agri-Food and Biosciences Institute, Hillsborough, United Kingdom,

${ }^{3}$ Agri-Food and Biosciences Institute, Belfast, United Kingdom Email: peter.kennedy@afbini.gov.uk

Introduction With increasing consumer awareness of meat quality and the relationship between dietary fat and the incidence of cardiovascular related disease, research has been undertaken to manipulate fatty acid (FA) profiles in beef. Scollan et al, (2006) found that legume based diets resulted in higher polyunsaturated fatty acid (PUFA) levels in intramuscular fat in beef steers than those offered grass silage. The aim of this experiment was to assess the effect of offering lupins/triticale, lupins/wheat, peas/oat wholecrop silage and grass silage to continental beef cattle on instrumental meat quality and FA composition.

Materials and methods Ninety continental cross steers $(555 \pm 41 \mathrm{~kg})$ were allocated to one of 10 dietary treatments in a 5 forage x 2 concentrate level experiment. The five forage diets offered were (1) perennial ryegrass based grass silage (PGS), (2) fescue/perennial ryegrass based grass silage (FGS), (3) lupins/triticale based silage combined with PGS 50:50 on a dry matter (DM) basis, (4) lupins/wheat based silage combined with PGS 50:50 on a DM basis, and (5) peas/oat wholecrop silage combined with PGS 50:50 on a DM basis. Each forage diet was offered ad libitum and supplemented with either 4 or $7 \mathrm{~kg}$ concentrates/head/day. A representation of steers from each forage and concentrate treatment were slaughtered over each of the 4 dates, ranging from 109 to 137 days on trial. Instrumental meat quality assessments (cooking loss, Warner Braztler shear force, meat colour) were carried out 7 days post mortem. FA analyses were undertaken on the longissimus dorsi obtained from the fore-rib joint using the direct methyl ester preparation method of O'Fallon et al. (2007) and capillary column gas chromatography. FA concentrations were expressed as g FA/100 g total FA. Data were analysed as a 5 forage $\mathrm{x} 2$ concentrate level factorial experiment with genotype, farm of origin and start weight included as covariates using GenStat REML.

Results The effect of these treatments on production and carcass characteristics was presented previously by Kennedy and Dawson (2009). Forage offered to continental finishing steers had no effect on FA composition of muscle (Table 1). Increasing concentrate level significantly $(\mathrm{P}<0.05)$ decreased concentration of $\alpha$-linolenic acid $(\mathrm{C} 18: 3 \mathrm{n}-3)$ but had no effect on total n-3 PUFA, n-6 PUFA, PUFA:SFA ratio and conjugated linoleic acid (CLA) level in muscle. Forage had no effect on instrumental meat quality. Increasing concentrate level offered from 4 to $7 \mathrm{~kg} / \mathrm{head} / \mathrm{day}$, increased $\mathrm{a}^{*}$ and $\mathrm{b}^{*}$ but had no effect on $\mathrm{L}^{*}$ of lean meat colour, cooking loss or Warner Braztler shear force (WBSF). An increase in $\mathrm{b}^{*}$ from lean meat offered a higher concentrate ratio to grass silage is in contrast to previous research (Cooke et al (2004). There were no statistically significant interactions between forage diet and concentrate level.

Table 1 Effect of forage type and concentrate level on instrumental meat quality and fatty acid composition of muscle (g FA/100 g total FA) from continental steers

\begin{tabular}{|c|c|c|c|c|c|c|c|c|c|c|c|}
\hline & \multicolumn{5}{|c|}{ Forage $(\mathrm{F})$} & \multicolumn{3}{|c|}{$\begin{array}{l}\text { Concentrate } \\
(\mathrm{kg} / \text { day })(\mathrm{C})\end{array}$} & \multirow[b]{2}{*}{ sed } & \multicolumn{2}{|c|}{ Significance } \\
\hline & $\mathrm{PGS}^{1}$ & FGS $^{2}$ & $\begin{array}{l}\text { Lupins/ } \\
\text { triticale }^{3}\end{array}$ & $\begin{array}{l}\text { Lupins/ } \\
\text { wheat }^{4}\end{array}$ & $\begin{array}{l}\text { Peas/ } \\
\text { Oat }^{5}\end{array}$ & sed & 4 & 7 & & $\mathrm{~F}$ & $\mathrm{C}$ \\
\hline \multicolumn{12}{|l|}{ Fatty acid composition } \\
\hline n-3 PUFA & 0.79 & 0.96 & 0.71 & 0.85 & 0.85 & 0.119 & 0.89 & 0.77 & 0.073 & NS & NS \\
\hline n-6 PUFA & 2.53 & 3.13 & 3.19 & 3.53 & 3.20 & 0.473 & 3.07 & 3.16 & 0.292 & NS & NS \\
\hline C18: 3n-3 & 0.60 & 0.74 & 0.61 & 0.64 & 0.60 & 0.079 & 0.70 & 0.58 & 0.049 & NS & $*$ \\
\hline PUFA:SFA & 0.07 & 0.08 & 0.08 & 0.09 & 0.08 & 0.012 & 0.08 & 0.08 & 0.008 & NS & NS \\
\hline Total CLA & 0.41 & 0.38 & 0.34 & 0.36 & 0.45 & 0.065 & 0.37 & 0.41 & 0.040 & NS & NS \\
\hline \multicolumn{12}{|l|}{ Instrumental meat quality } \\
\hline Cooking loss $(\mathrm{g} / \mathrm{kg})$ & 27.4 & 26.3 & 27.6 & 27.7 & 27.6 & 0.62 & 27.5 & 27.1 & 0.39 & NS & NS \\
\hline WBSF $\left(\mathrm{kg} / \mathrm{cm}^{2}\right)^{\gamma}$ & 4.88 & 4.34 & 4.55 & 4.79 & 4.61 & 0.217 & 4.51 & 4.76 & 0.136 & NS & NS \\
\hline L* (lightness) & 40.5 & 40.7 & 39.9 & 41.7 & 40.3 & 1.32 & 41.2 & 40.0 & 0.83 & NS & NS \\
\hline $\mathrm{a}^{*}($ redness $)$ & 25.6 & 25.6 & 25.0 & 24.3 & 26.0 & 1.14 & 24.5 & 26.1 & 1.61 & NS & $*$ \\
\hline b* (yellowness) & 17.1 & 16.9 & 15.8 & 16.3 & 17.6 & 0.89 & 16.2 & 17.3 & 0.56 & NS & $*$ \\
\hline
\end{tabular}

Treatments 1 to 5 as described in methods, $* \mathrm{P}<0.05$, NS not statistically significant ( $\mathrm{P}>0.05$ )

Conclusion Finishing beef cattle on legume/cereal wholecrop silage had no effect on muscle fatty acid composition or instrumental meat quality relative to grass silage based diets. An increased level of concentrate supplementation decreased C18:3n-3 proportion and increased redness $\left(a^{*}\right)$ and yellowness $\left(b^{*}\right)$ of the meat.

Acknowledgements Funding from EBLEX, HYBU CIG CYMRU, QMS, AgriSearch and DARDNI is gratefully acknowledged.

\section{References}

Cooke, D.W., Monahan, F.J, Brophy P.O. and Boland, M P 2004 Irish Journal of Agricultural and Food Research 43, $201-216$.

Kennedy, P C and Dawson, L E R 2009, Proceedings of the $9^{\text {th }}$ British Grassland Society Conference, p 11-12.

O'Fallon, J. V., Busboom, J. R., Nelson, M. L. and Gaskins, C. T. 2007 Journal of Animal Science, 85, 1511-1521

Scollan,. N. D., et al. 2006 Proceedings of BSAS, p23. 